\title{
Influence of Camera Setting on Vehicle-to-Vehicle VLC Employing Undersampled Phase Shift On-Off Keying
}

\author{
Stanislav VITEK ${ }^{1}$, Jiri LIBICH ${ }^{1}$, Pengfei LUO ${ }^{2}$, Stanislav ZVANOVEC ${ }^{1}$, Zabih GHASSEMLOOY ${ }^{3}$, \\ Navid Bani HASSAN ${ }^{3}$ \\ ${ }^{1}$ Faculty of Electrical Engineering, Czech Technical University in Prague, Technická 2, Prague, 166 28, Czech Republic \\ ${ }^{2}$ Research Department of HiSilicon, Huawei Technologies Co., Ltd, Beijing, 100085, China \\ ${ }^{3}$ Faculty of Engineering and Environment, Northumbria University, Newcastle upon Tyne, NE1 8ST, United Kingdom
}

viteks@fel.cvut.cz, oliver.luo@hisilicon.com,xzvanove@fel.cvut.cz, z.ghassemlooy@northumbria.ac.uk, navid.hassan@northumbria.ac.uk

Submitted February 16, 2017 / Accepted August 4, 2017

\begin{abstract}
This paper focuses on the performance analysis of a camera-based vehicle-to-vehicle visible light communication system employing undersampled phase shift on-off keying modulation under interference scenario. Two Nissan Qashqai front lights with daylight running light emitting diodes based lamps are used for communications. The bit error rate (BER) performance of the proposed system is experimentally measured for a transmission span up to $24 \mathrm{~m}$ focusing mostly on the side interference due to reflections. Based on experimental data we demonstrate reduction of the system performance due to the side reflection and illumination of the detector by other light sources which have to be taken into account during further data processing. We provide with further statistics for particular shutter speed and transmitter power setting and discuss BER improvement especially to meet FEC via the method of the adaptive region of interest.
\end{abstract}

\section{Keywords}

Visible Light Communication, Vehicle-to-Vehicle communication, undersampled phase shift on-off keying, low bitrate, camera

\section{Introduction}

Today's dense road traffics in combination with relatively fast cars require additional improvements in the car and road safety. Transportation has become an essential part of everyday life. New safety systems have been developed thanks to newly emerged technologies such as anti-collision and intelligent transport systems (ITS) [1]. The widespread deployment of ITS will achieve a high level of continuous traffic flow, safety, and a lower carbon footprint at a global level. Vehicular networking (VN) is an essential component of ITS, which offers vehicle-to-vehicle (V2V) and vehicleto-infrastructure (V2I) communications. The information gathered via V2V and V2I provides the drivers with realtime information on the traffic and road conditions including collisions, congestion, surface condition, traffic signal violations, emergency brakes, etc. [2], [3] Furthermore, such information can be used by the local transport departments to develop efficient traffic applications (e.g., speed advisory, platooning) or by automotive manufacturers to develop safety applications (like collision avoidance). To achieve this, ITS have taken advantages of the information and communication technologies (ICT) to assist the vehicle users. The most widely adopted ICT are based on the short-range radio frequency wireless technologies (i.e., ultra-high frequency and very high-frequency) employing IEEE 802.11 protocols. However, longer range communications have been proposed based on worldwide interoperability for microwave access (WiMAX) IEEE 802.16, global system for mobile Communications $(3 \mathrm{G}, 4 \mathrm{G})$ requiring extensive and very costly infrastructure deployment compared to the shorter range system [4], [5].

ITS has also been considering the use of visible light communications (VLC) [6] by way of exploiting the potential of light-emitting diode (LED) based lighting fixtures in both vehicles and roadsides (i.e., street lamps, traffic lights, car lights). This approach will lead in the long run to reduced cost, greener environment and the release of highly need RF frequency spectrum for other applications. VLC (in the spectrum range of 380 to $780 \mathrm{~nm}$ ) has attracted the attention of many researchers in the last decade with dedicated IEEE 802.15.7r1 standard [7], which defines network architectures, physical and medium access control (MAC) layers. The LED light sources adapted are characterized mostly by their lower power consumption, relatively higher energy efficiency, switching capability and much wider bandwidth compared to the incandescent and fluorescent lights. Hence LEDs are not only suitable for illumination but also for data transmission, and sensing as well as for very precise localization (e.g. in [8] reported precision $<1.7 \mathrm{~cm}$ for indoor navigation), which cannot be achieved in the RF domain. 


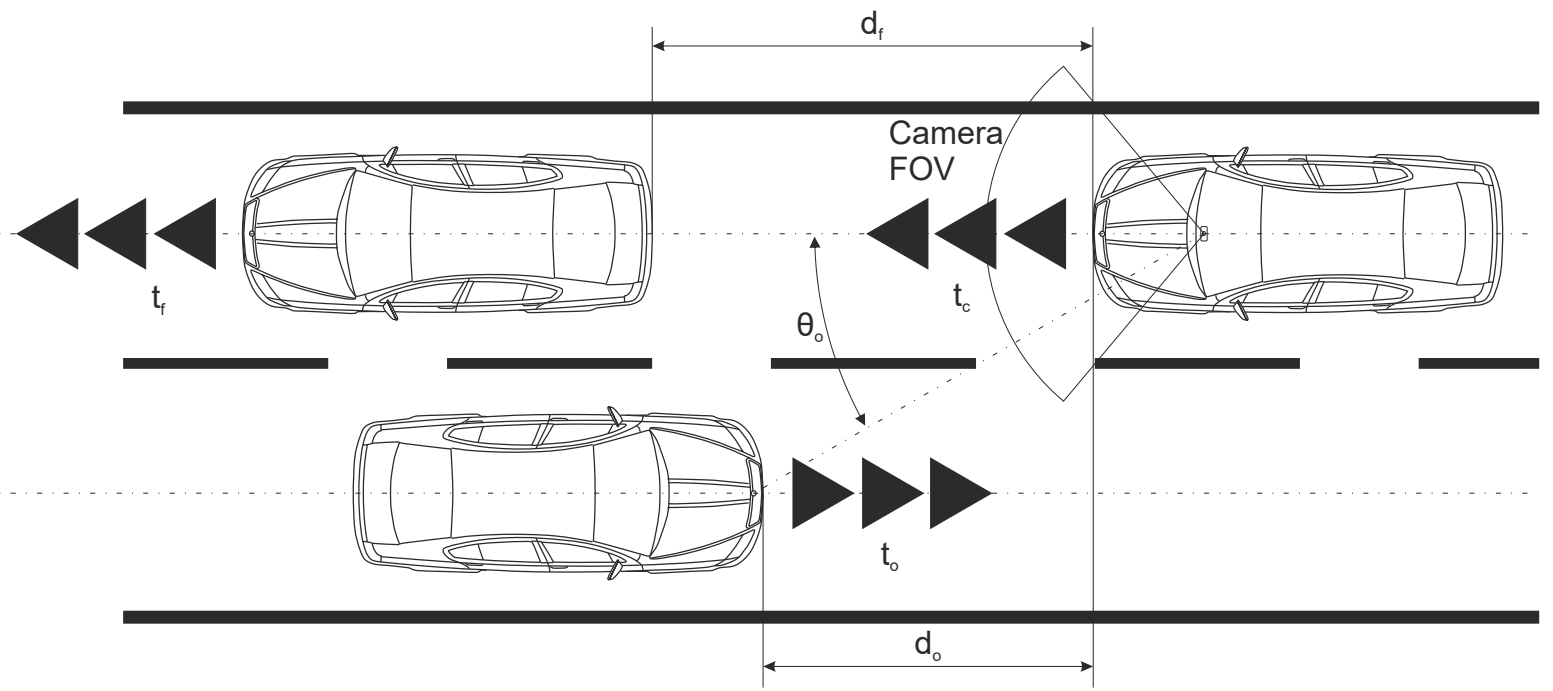

Fig. 1. Typical road traffic situation for a two-lane road.

VLC systems employ LEDs as the transmitting source and a photodiode (PD) or a camera sensor (CS) as the receiving module. Almost all modern vehicles have already LED-based head and brake lights, and indicator lights, thus being possible for the concept of vehicular VLC (VVLC) as a new cost-effective way to implement V2V and V2I communications [9-13]. Furthermore, LED-based roadside units can also be used for both signaling and broadcasting safetyrelated information to vehicles on the road.

Several test use-cases and experimental results have been published for VVLC networks consisting of onboard units, vehicles, and road side units (i.e., traffic lights, street lamps, digital signage, etc.) [15], [14]. Recent studies reported on V2V communications use either PDs or a CS to detect oncoming vehicles and subsequently control the illumination pattern of the head lights to avoid the glaring. Furthermore, the camera concept can also be used as a receiver (Rx) as part of the VLC based ITS [16], [17], known as the optical camera communications (OCC) [18]. VLC systems based on the LED transmitter (Tx) and a camera based $\mathrm{Rx}$ were proposed for automotive applications in [19], where a signal reception experiments were performed under laboratory conditions with fixed positions and then within outdoor environment with changed distance of the receiving camera Rxs with up to a $15 \mathrm{Mb} /$ pixel/s error-free performace. In [20], it was shown that under the driving condition the Rx could detect and accurately track an LED Tx array with an errorfree communication over a transmission range of $25-80 \mathrm{~m}$. Contrary to a typical VLC communications scenario (with a data source, driver, Tx, PD-based Rx, and processing units), the camera-based VLC system can receive and separate multiple light sources within its field of view (FOV) and extract the information using image sequence processing. This detection technique also offers a unique feature, for example, utilization of the multiple-input and multiple-output (MIMO) capabilities supporting parallel data transmission [21].
Nowadays, many new cars are equipped with a built-in camera for data recording and detection of road condition for safety reason and headlight illumination control. Such a camera can also be used for data communications as well as sensing as part of VLC based ITS. Because of the low frame rate of standard low-cost cameras, OCC only offers a low data rate, typically tens of bits per second, which is sufficient for traffic related information transmission as part of ITS. With respect to such a low data, communication will have a form of periodic broadcast of the short messages, such as hazardous location warning, traffic signal violation warning, etc. This paper is focused on the experimental verification of the camera setting on V2V VLC employing the undersampled phase shift on-off keying modulation scheme, which uses two square wave carriers at the same frequency, with the same amplitude and opposite phases to represent bits 0 and 1 .

The paper is organized as follows. At first, the main concept of the V2V system model is introduced in Sec. 2 taking into consideration typical V2V VLC scenario and the main drawbacks that have to be considered in the experimental part. We have focused especially on the side interference reflection. Section 3 outlines setup of the experimental campaign. The results from the OCC VLC system measurement including the bit error rate (BER) performance for different camera parameter settings are given in Sec. 4. Finally, the conclusion is discussed in Sec. 5.

\section{System Scenario}

Figure 1 shows a typical two line road case where vehicles are communicating with each other using OCC VLC [22]. The cars have two cameras to detect the cars at the front as well as the oncoming vehicles. In detecting vehicles, the beam direction and shape can be adjusted to 


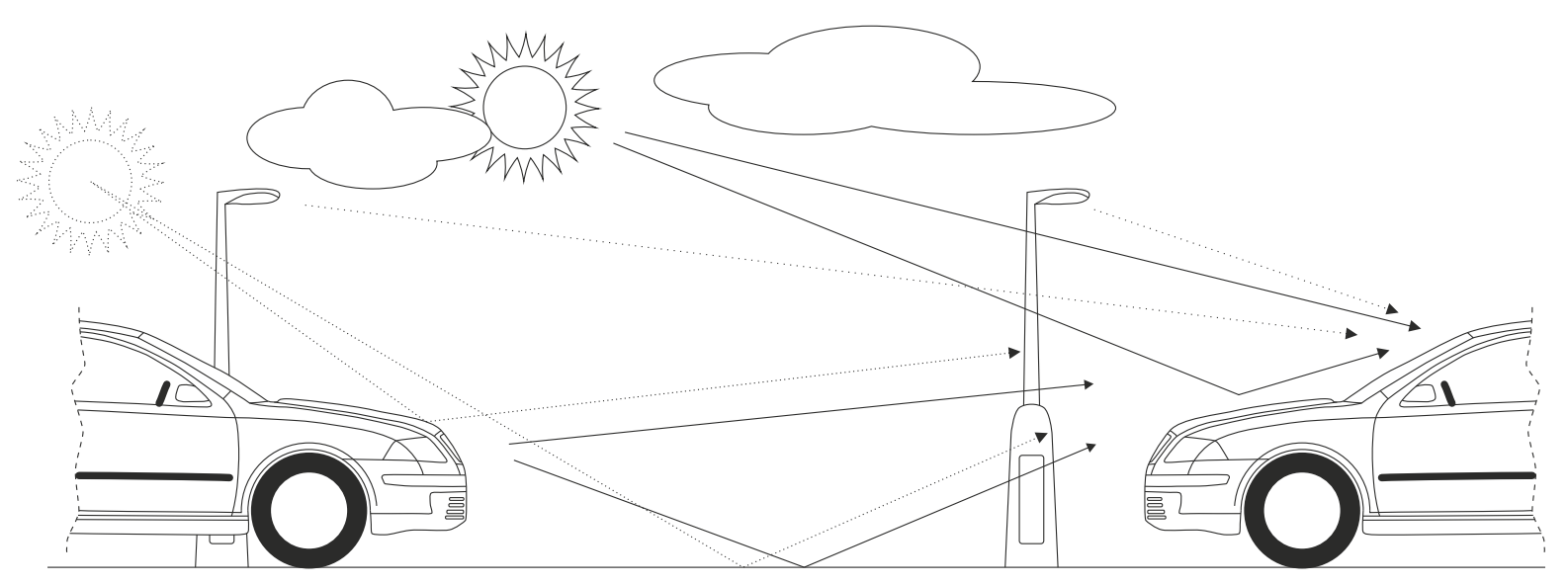

Fig. 2. A C2C VLC link with possible noise sources.

avoid dazzling of other drivers. The VLC communication link is used to transmit traffic related information between the cars.

Figure 2 depicts the both line of sight (LOS) and NLOS (non-LOS) paths including as well interference due to lights from the Sun, other vehicles, street light, etc. which can reach the camera based Rx positioned within the cars. When a light beam illuminates a surface, it results in reflection, transmission, and absorption from the road surface [23]. Contrary to the PD-based Rx, which combines the light rays from LOS and NLOS paths, the camera-based Rx can spatially distinguish each reflected and non-reflected light sources, and process them separately. The reflection from the road surface not only depends on the tarmac road materials but also on the weather conditions. A dry surface road has a diffuse characteristic where an incident light ray is reflected at many angles with some reaching the $\mathrm{Rx}$, whereas under the wet road surface condition the reflection is specular similar to a mirror (including polarization properties) [24].

The received optical power from a single head light can be expressed in the case of the LOS propagation by [25]:

$$
P_{\mathrm{LOS}}= \begin{cases}\frac{I_{\mathrm{R}} \cdot A_{r} \cdot \cos \left(\Theta_{\mathrm{BLOS}}\right)}{L E R \cdot d_{\mathrm{LOS}}^{2}}, & 0 \leq \Theta_{\mathrm{BLOS}} \leq \Psi, \\ 0, & \Theta_{\mathrm{BLOS}}>\Psi\end{cases}
$$

where $I_{\mathrm{R}}$ is luminous intensity of the car light in a particular direction, $\Theta_{\mathrm{BLOS}}$ denotes the angle between the PD surface normal and the incident Line-of-sight direction, $d_{\mathrm{LOS}}$ is the direct distance between the source and detector, $A_{r}$ and $\Psi$ are active surface area and field of view of the PD, respectively, and $L E R$ stands for the luminous efficiency of radiation.

For the NLOS propagation path between the source and detector the received optical power is given as

$$
\begin{gathered}
P_{\mathrm{NLOS}}= \begin{cases}\iint \mathrm{d} P_{\mathrm{NLOS}} \mathrm{d} S, & 0 \leq \Theta_{\mathrm{BNLOS}} \leq \Psi, \\
0, & \Theta_{\mathrm{BNLOS}}>\Psi\end{cases} \\
\mathrm{d} P_{\mathrm{NLOS}}=\frac{d I_{\mathrm{R}} \cdot A_{r} \cdot \cos \left(\Theta_{\mathrm{BLOS}}\right)}{L E R \cdot d_{\mathrm{NLOS}}^{2}}
\end{gathered}
$$

where $d_{\text {NLOS }}$ is the NLOS path length, $\Theta_{\text {NLOS }}$ denotes the angle between the road surface or surrounding reflecting object and the PD surface normal, and $S$ is the entire area of the surface that has been illuminated by the source.

\subsection{Undersampled Phase Shift On-Off Keying}

VLC links based on camera Rxs typicaly use undersampled frequency shift on-off keying (UFSOOK) [26], employing two square wave based carrier signals at frequencies of $f_{\mathrm{c} 0}, f_{\mathrm{c} 1}$ to represent bits 0 and 1 , respectively. Additionally, there is a square wave signal with higher frequency

$$
f_{\mathrm{cFH}}>\frac{1}{\text { shutter speed }},
$$

which represents the intermediate state or also known as the frame header $(\mathrm{FH})$.

In UFSOOK, aliasing is intentionally introduced, and the original data can be decoded from the sampled results. Note that at the Rx sampling is done at the camera frame rate $f_{\mathrm{c}}$. With a camera based $\mathrm{Rx}$, a number of frames per second (fps) with a fixed or variable exposure time can be captured. Normally, for commercial cameras, $f_{\mathrm{c}} \leq 60 \mathrm{fps}$ to support data transmission with no flickering. Therefore, the baseband data should be modulated at higher frequencies, and the modulated signal must conform to the flicker-free illumination requirement.

Undersampled phase shift on-off keying (UPSOOK) [27], [28] uses opposite phases to represent bits 0 and 1 and scheme supports flicker-free OCC. Note that the critical flicker-fusion frequency (CFF) of an average human eye $f_{\text {eye }}$ is about $100 \mathrm{~Hz}$ [29] and CFF of the camera $f_{\text {ca }}$ is given by

$$
f_{\text {ca }}=\frac{1}{\text { shutter speed }} \text {. }
$$

In UPSOOK, a square wave subcarrier with a frequency

$$
f_{\mathrm{s}}=n \cdot f_{\mathrm{c}}, \quad f_{\text {eye }}<f_{\mathrm{s}}<f_{\text {ca }}
$$




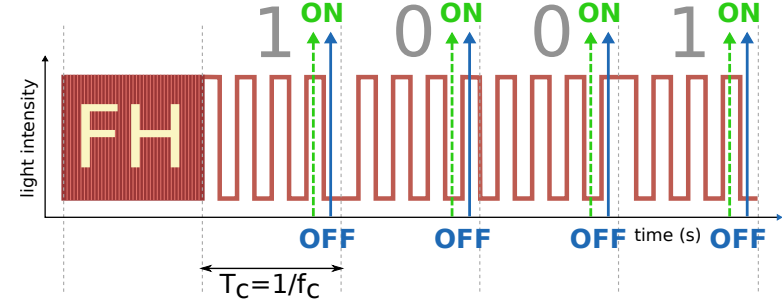

Fig. 3. UPSOOK waveform and two possible undersampled cases for the same transmitted signal.

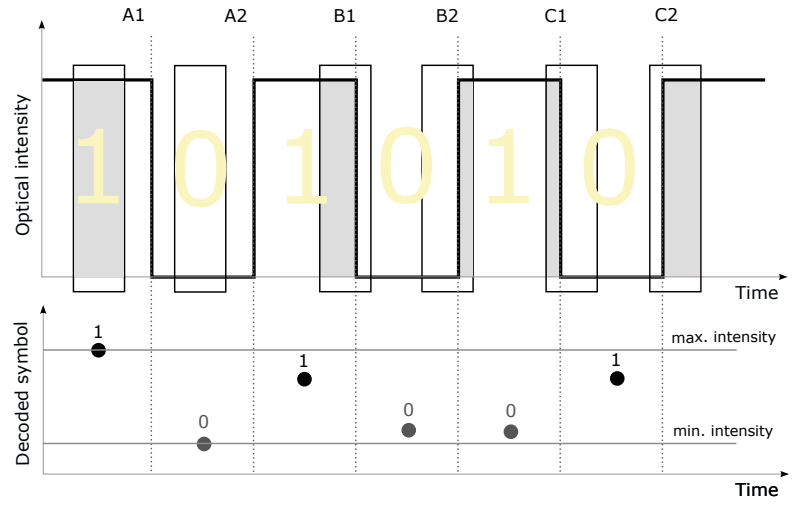

Fig. 4. Illustration of sampling in UPSOOK based OCC VLC.

where $n$ is an integer with a typical value higher than two. Figure 3 shows an example of the UPSOOK modulated signal with a $\mathrm{FH}$. The $\mathrm{FH}$ is a $50 \%$ duty cycle square wave with a frequency of $10 \mathrm{kHz}$, which is much higher than $f_{\text {ca. }}$. For the modulated bits 1 (mark) and 0 (space) the symbol duration $T_{\mathrm{c}}=1 / f_{\mathrm{c}}$ to ensure that the symbol rate $R_{S}=1 / T_{\mathrm{c}}$ and $f_{\mathrm{c}}$ are the same. Therefore, the camera can correctly undersample the bit stream at each sampling strobe.

The UPSOOK signal can be expressed as

$$
s(t)=\left\lceil\cos \left(2 \pi f_{\mathrm{c}} t+\theta_{n}\right)\right\rceil, \quad 0<t \leq T_{\mathrm{c}}
$$

where $\lceil$.$\rceil denotes a square wave with the phase \theta_{n}$ modulated by an input data $a_{n}$ given by

$$
\theta_{n}= \begin{cases}0^{\circ}, & a_{n}=1, \\ 180^{\circ}, & a_{n}=0 .\end{cases}
$$

Since the sampling phase of the camera at the $\mathrm{Rx}$ is generally not controlled, there will be a random phase difference between Tx and the camera. Figure 3 illustrates two possible undersampled scenarios obtained by two groups of sampling strobes: green (dashed lines) and blue (solid lines) strobes. Ideally, the sampling points should be at the center of each symbol. Nevertheless, owing to the asynchronous transmission, the sampling point is randomly shifted within the symbol period.

Note, the sampling points of A1 and A2, and B1 and $\mathrm{B} 2$ results in normal and shorter sampling intervals, respectively as shown in Fig. 4. The sampling points of $\mathrm{C} 1$ and $\mathrm{C} 2$ illustrate the extreme case where samplings are right at the transition edges of the pulse, thus may result in wrong decision making between the logic levels of 0 and 1 . Therefore, this effect together with the integration time of the camera sensor will result in reduced signal to noise ratio (SNR). At higher shutter speed the integration time is shorter and therefore the probability that the integration window will be between two symbols is rather low. However, the SNR can be improved by increasing the integration time but at the cost of the integration window spreading over two symbols. In the extreme case, when the center of the integration window is at the edge of a symbol, then the probability of 1 being read as 0 and vice versa increases, thus leading to higher BER.

\section{Experimental Measurement}

We have carried out experimental verification of the proposed OCC VLC system composed of two LED-based daylight running light (DRL) modules and a camera-based $\mathrm{Rx}$ in an indoor environment. The focus of our investigation was mostly on the non-LOS reflected paths to mimic the outdoor condition. A pseudorandom binary sequence of length of 100 symbols was generated and subsequently converted into the UPSOOK format [27] (i.e. total length of the frame is 102 symbols including $\mathrm{FH}$ and one symbol representing symbol 1 following FH). A Tektronix AFG3252C arbitrary waveform generator was used to generate the UPSOOK signal for intensity modulation (IM) of two LED headlights for Nissan Qashqai vehicle, see Fig. 5.

Based on the results published in [30], which outlines that the typical duration of V2V VLC link is in the order of seconds, we set the transmission rate to $50 \mathrm{fps}$. It means that transmission of a single frame takes approximately 2 second. An 18-megapixel Canon EOS 100D camera with a shutter speed ranging from $1 / 4000$ to 30 seconds was used to capture and record the IM LED light sources, see Fig. 6. The captured video stream was stored in the camera as MOV files with a resolution of $720 \mathrm{p}$. The focal length of the camera was set to $18 \mathrm{~mm}$. The transmission link length was set within the range of $2 \mathrm{~m}$ to $26 \mathrm{~m}$, with the step of $6 \mathrm{~m}$. Note, at $30 \mathrm{mph}$ the thinking distance, the braking distance and the average distance to stop are around 9, 14 and $23 \mathrm{~m}$, respectively. Two different shutter speeds were used, 1/800 and 1/4000.

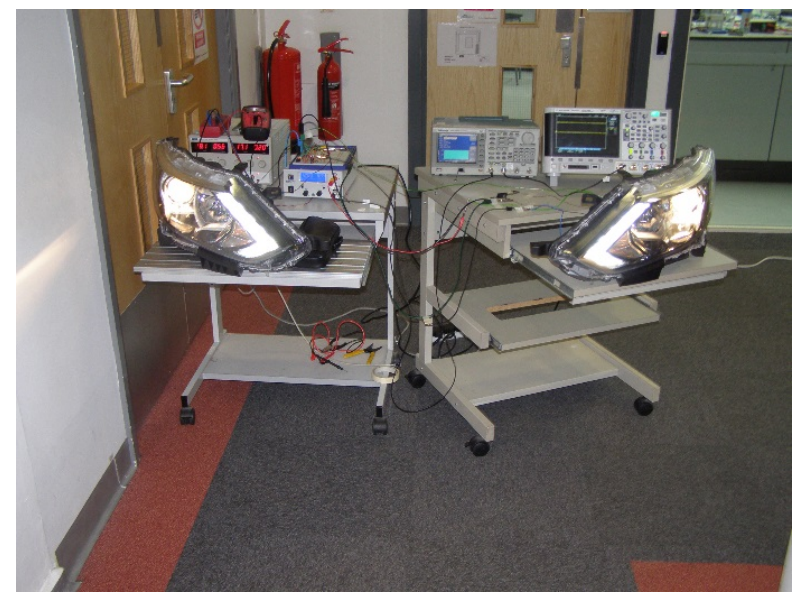

Fig. 5. Experimental setup: the Tx. 


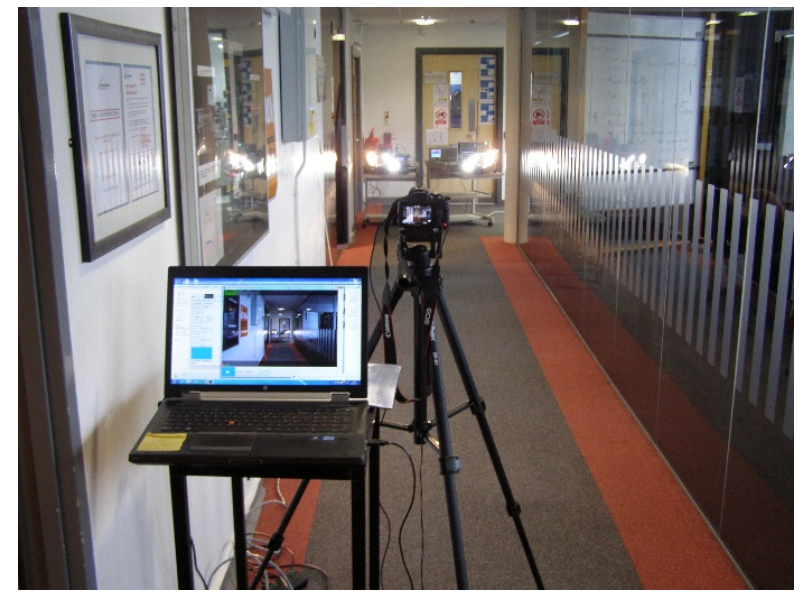

Fig. 6. Experimental setup: the Rx.

The frequency of the square wave carrier was chosen $200 \mathrm{~Hz}$ (i.e. $n=4$ in (6)). The data stream was packaged into data frames. Each frame began with a header consisted of a symbol formed by a $10 \mathrm{kHz}$ signal (camera sees this signal as a half of the peak to peak optical power) followed by a single bit of logical 1 to correct the potential unsynchronized phase between the Tx and the Rx.

In [27], a system utilizing a mapping of bits via two LED Txs to increase the data rate was reported. In our experimental work, we have used both DRLs to transmit the same data. During tests, we analyzed OCC VLC result when the front light low beam (LB, in parallel used only for illumination, not carrying VLC) turned on or off in order to influence the VLC link performance. We modulated the light intensity by applying two sets of current, $200 \mathrm{~mA}$ and $600 \mathrm{~mA}$.

\section{Results}

We considered two types of detection with the camera based Rx:

(i) camera emulates PD Rx - averaging entire camera FOV (see Fig. 7a) into one value. Such a detector does not need any further image processing, but all reflections and noises are integrated in the reception and affect the quality of transmission significantly.

(ii) The adaptive region of interest (ROI) - adaptively capturing always one Tx area (ROI for a distance of $2 \mathrm{~m}$ see Fig. 7b) - when a detector needs a tracking algorithm in case of moving cars and real-time image-processing.

In both cases as mentioned above, the off-line data processing provides the simple sum of pixel values in the selected ROI (or whole frame). The frames are then converted from RGB color space to grayscale. Note that FH were manually identified and also thresholds to distinguish between symbol 0 and symbol 1 were founded separately for particular video sequence.

Figure 8 then illustrated the example of decoded UPSOOK waveform with cropped captured frames. Although the quality of this link is perfect, one may see the effect of the

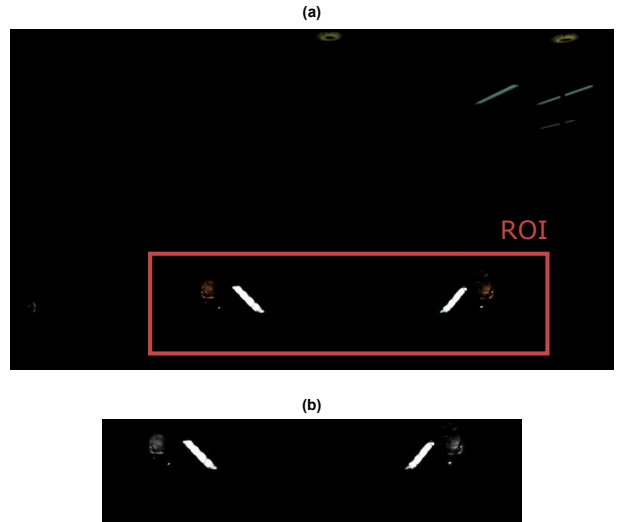

Fig. 7. Example of image data. (a) Whole camera FOV. (a) ROI covers both left and right LED headlights.
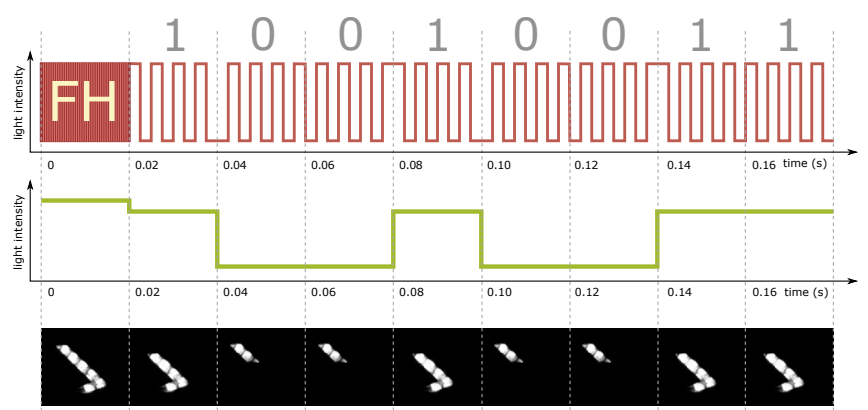

Fig. 8. Example of decoded data. Shutter-speed 1/4000, LB off. LED current $600 \mathrm{~mA}$.

phase shift between the sampling strobe and the waveform, which are labeled as B1 and B2, respectively, in Fig. 4.

From data processing regarding BER statistics, it was observed that most of the errors were due to a phase shift of sampling time and symbol position. Contrary to the emulating PD (whole picture averaging), the link length was not a limiting factor for data transmission in the case of adaptive ROI as can be demonstrated in Fig. 9 and 10. Because of a very low-speed data rate, it is not uncommon to obtain relatively high values of BER in contrast to conventional telecommunication links, especially when a link connection lasts only a few seconds. The primary source of the increased BER due to the reflection of the low beam especially for the case of shutter speed of $1 / 800$ where the BER is above the forward error correction (FEC) $7 \%$ BER limit of $3.8 \cdot 10^{3}$ [31]. This can be clearly seen from Fig. 9a, where values of BER in such case do not meet FEC limit for distances longer than (i) $8 \mathrm{~m}$ for the shutter-speed of 1/800 and the LED current of $200 \mathrm{~mA}$ with LB is off; (ii) $14 \mathrm{~m}$ for the shutter speed of $1 / 800$ and the LED current of $600 \mathrm{~mA}$; (iii) $8 \mathrm{~m}$ for the shutterspeed of $1 / 800$ and the LED current of $200 \mathrm{~mA}$; (iv) $8 \mathrm{~m}$ for the shutter-speed of $1 / 4000$ and the LED current of $200 \mathrm{~mA}$ when LB is off. BER does not meet the FEC limit for the shutter-speed of 1/800 and the LED current of $200 \mathrm{~mA}$ when LB is on. This has to be taken into account in further preprocessing of the received data. It is also evident, that quality of the link is significantly worsen when lower LED current 

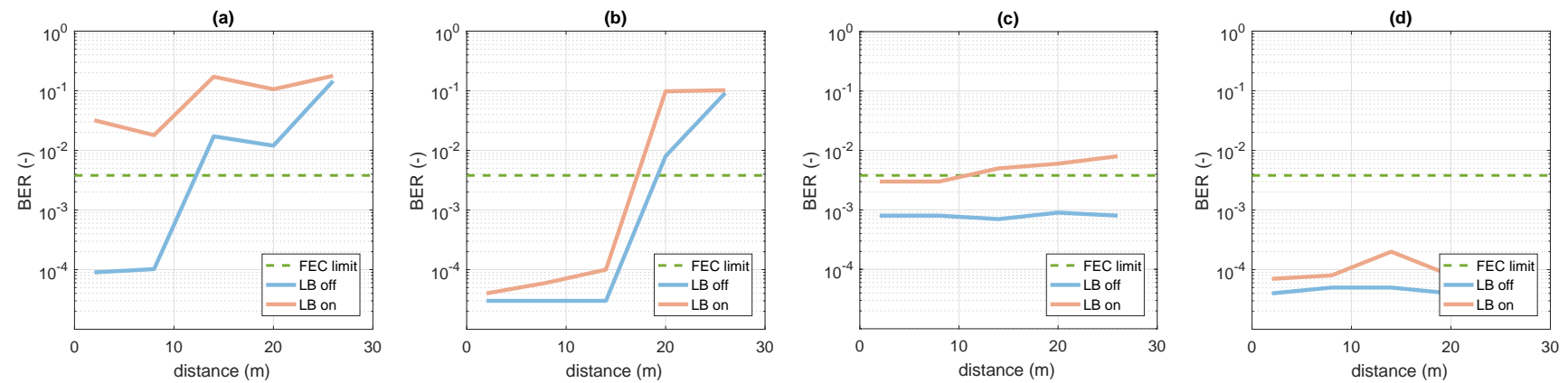

Fig. 9. V2V OCC BER as a function of the distance from Tx for camera Rx emulates PD. a) Shutter-speed 1/800. LED current $200 \mathrm{~mA}$. b) Shutter-speed 1/800. LED current $600 \mathrm{~mA}$. c) Shutter-speed 1/4000. LED current $200 \mathrm{~mA}$. d) Shutter-speed 1/4000. LED current $600 \mathrm{~mA}$.
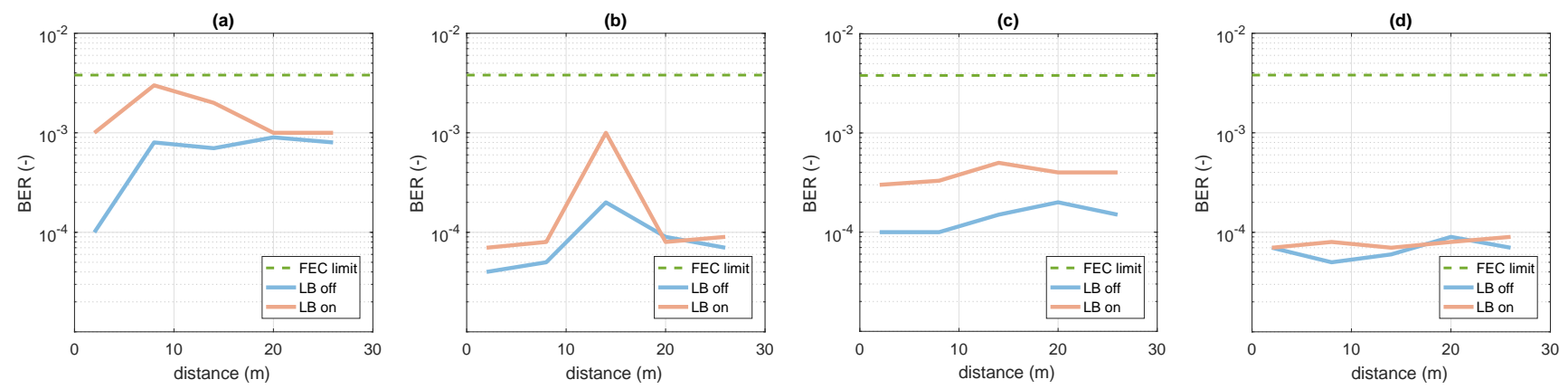

Fig. 10. V2V OCC BER as a function of the distance from Tx for camera Rx uses adaptive ROI. a) Shutter-speed 1/800. LED current $200 \mathrm{~mA}$. b) Shutter-speed 1/800. LED current $600 \mathrm{~mA}$. c) Shutter-speed 1/4000. LED current $200 \mathrm{~mA}$. d) Shutter-speed 1/4000. LED current $600 \mathrm{~mA}$

(a)

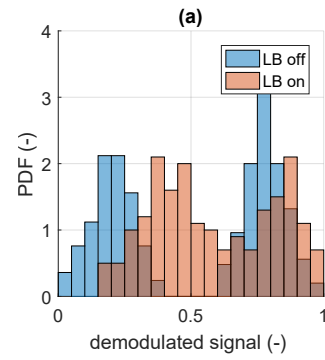

(b)

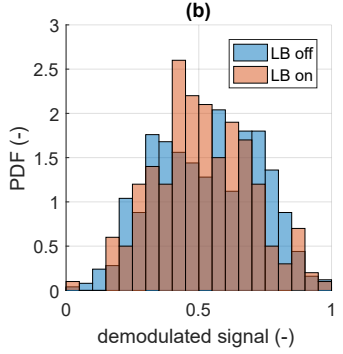

(c)

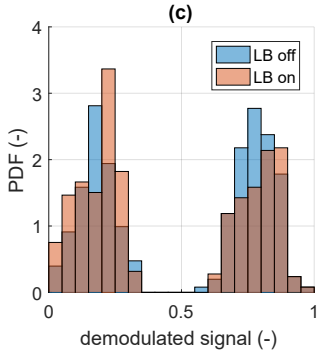

(d)

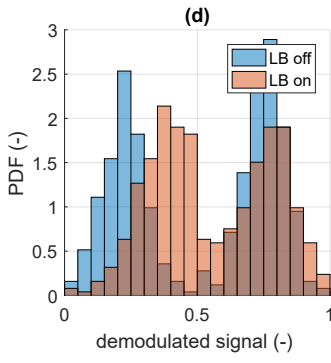

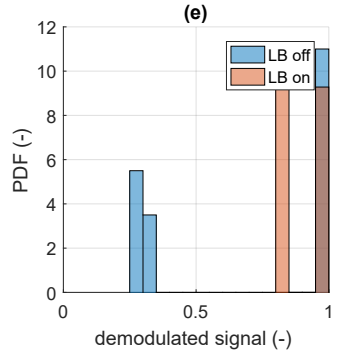

Fig. 11. Examples of received data PDF. (a) Entire FOV. LED current $200 \mathrm{~mA}$. Shutter-speed 1/800. Distance $2 \mathrm{~m}$. (b) Entire FOV. LED current 200 mA. Shutter-speed 1/800. Distance 26 m. (c) Entire FOV. LED current $600 \mathrm{~mA}$. Shutter-speed 1/800. Distance $2 \mathrm{~m}$. (d) Entire FOV. LED current $600 \mathrm{~mA}$. Shutter-speed 1/800. Distance $26 \mathrm{~m}$. (e) Selected ROI. LED current $600 \mathrm{~mA}$. Shutter-speed 1/800. Distance $26 \mathrm{~m}$.

is used. Faster shutter speed reduces BER as an be seen in Fig. 9b. Considerable improvement is achieved when ROI implemented - as shown in Fig. 10 BER has been reduced bellow the FEC limit for both shutter speeds thus providing seamless communications over the entire transmission span.

The camera based Rx needs to consider several issues that influence link performance: (i) when recording a video stream, the entire picture may not be captured at the same time (i.e., rolling shutter effect) (ii) asynchronous data transmission. The latter is when the camera captures the image exactly at the transition time of the symbol; it is not possible to distinguish the symbols 0 and 1 because of the integrator functionality of the image sensor as described above. For the sake of completeness, Fig. 11 displays examples of the probability density functions (PDF) of the decoded data, which clearly demonstrating how particular effect results in overlaping of detected 0 and 1 symbols. The histograms show PDF calculated from ten consecutive frames, i.e. 1020 symbols.
The values obtained as a sum of all pixels in the grayscale image were normalized relative to the highest value in that sequence. While Figures $11 \mathrm{a}$ and $11 \mathrm{~b}$ illustrates how the distance between the Tx and the Rx affects distinctiveness of symbols, Figures 11c and 11d show the role of the LED current. Figure 11e then shows the impact of the ROI.

\section{Conclusion}

Even though the car to car communications have been considered as new ways to improve road safety and several papers have been published recently a very limited number of papers have been devoted to the influence of the camera setting on the optical camera communications. Based on the experimental campaign employing common camera as part of the OCC system we tested adaptable low beam and surrounding reflection impact on the system performance. We have realized a VLC link to experimentally evaluate perfor- 
mance over a distance up to $24 \mathrm{~m}$ under several scenarios. It is evident that proper setting of the shutter speed and detected picture processing can result in BER being above the FEC limit in case of the low beam interference. These aspects have to be taken into account since they could considerably impact transmission of the messages between two moving vehicles when there are only short timeslots available.

\section{Acknowledgments}

The research is supported by the European social fund within the framework of the project CZ.1.07/2.3.00/30.0034. The joint research was supported by the EU COST ICT Action IC 1101 and by the Grant Agency of the CTU in Prague, grant no. SGS14/190/OHK3/3T/13.

\section{References}

[1] NASIM, R., KASSLER, A. Distributed architectures for intelligent transport systems: A survey. In Proceedings of the Second Symposium on Network Cloud Computing and Applications (NCCA). 2012, p. 130-136. DOI: $10.1109 /$ NCCA.2012.15

[2] CAVENEY, D. Cooperative vehicular safety applications. IEEE Control Systems, 2010, vol. 30, no. 4, p. 38-53. DOI: 10.1109/MCS.2010.937003

[3] WANG, Q., FAN, P., LETAIEF, K. B. On the joint V2I and V2V scheduling for cooperative VANETs with network coding. IEEE Transactions on Vehicular Technology, 2012, vol. 61, no. 1, p. 62-73. DOI: $10.1109 /$ TVT.2011.2167249

[4] MORGAN, Y. L. Notes on DSRC \& WAVE standards suite: Its architecture, design, and characteristics. IEEE Communications Surveys \& Tutorials, 2010, vol. 12, no. 4, p. 504-518. DOI: $10.1109 /$ SURV.2010.033010.00024

[5] LIBICH, J., ZVANOVEC, S., MUDROCH, M. Mitigation of timespatial influence in free-space optical networks utilizing route diversity. In Proceedings of the SPIE LASE International Society for Optics and Photonics. 2012, vol. 8246, p. 1-6. DOI: 10.1117/12.907928

[6] GHASSEMLOOY, Z., ALVES, L. N., ZVANOVEC, S., et al. Visible Light Communications Theory and Applications. CRC Press, 2017. ISBN: 9781498767538

[7] JUNGNICKEL, V., et al. A European view on the next generation optical wireless communication standard. In Proceedings of the IEEE Conference on Standards for Communications and Networking (CSCN). 2015, p. 106-111. DOI: 10.1109/CSCN.2015.7390429

[8] LUO, P., et al. Experimental demonstration of an indoor visible light communication positioning system using dual-tone multifrequency technique. In Proceedings of the 3rd International Workshop in Optical Wireless Communications (IWOW). 2014, p. 55-59. DOI: 10.1109/IWOW.2014.6950776

[9] LEE, S. J., et al. Evaluation of visible light communication channel delay profiles for automotive applications. EURASIP Journal on Wireless Communications and Networking, 2012, vol. 2012, no. 1. DOI: $10.1186 / 1687-1499-2012-370$

[10] YU, S. H., et al. Smart automotive lighting for vehicle safety. IEEE Communications Magazine, 2013, vol. 51, no. 12, p. 50-59. DOI: 10.1109/MCOM.2013.6685757
[11] YOU, S. H., et al. Demo: Visible light communications for scooter safety. In Proceedings of the 11th Annual International Conference on Mobile Systems, Applications, and Services (ACM). 2013, p. 509-510. DOI: 10.1145/2462456.2465707

[12] LIU, C. B., SADEGHI, B., KNIGHTLY, E. W. Enabling vehicular visible light communication (V2LC) networks. In Proceedings of the Eighth ACM International Workshop on Vehicular Inter-Networking. 2011, p. 41-50. DOI: 10.1145/2030698.2030705

[13] CAILEAN, A., et al. Visible light communications: Application to cooperation between vehicles and road infrastructures. In Proceedings of the IEEE Intelligent Vehicles Symposium (IV). 2012, p. 1055-1059. DOI: 10.1109/IVS.2012.6232225

[14] YAMAZATO, T., et al. Image-sensor-based visible light communication for automotive applications. IEEE Communications Magazine, 2014, vol. 52, no. 7, p. 88-97. DOI: 10.1109/MCOM.2014.6852088

[15] YAMAZATO, T., et al. Vehicle Motion and Pixel Illumination Modeling for Image Sensor Based Visible Light Communication. IEEE Journal on Selected Areas in Communications, 2015, vol. 33, no. 9, p. 1793-1805. DOI: 10.1109/JSAC.2015.2432511

[16] BELLE, A., et al. Development of IEEE 802.15.7 based ITS services using low cost embedded systems. In Proceedings of the 13th International Conference on ITS Telecommunications (ITST). 2013, p. 419-425. DOI: 10.1109/ITST.2013.6685583

[17] SAHA, N., et al. Survey on optical camera communications: Challenges and opportunities. IET Optoelectronics, 2015, vol. 9, no. 5, p. 172-183. DOI: 10.1049/iet-opt.2014.0151

[18] LUO, P., et al. Experimental demonstration of a 1024-QAM optical camera communication system. IEEE Photonics Technology Letters, 2016, vol. 28, no. 2, p. 139-142. DOI: 10.1109/LPT.2015.2487544

[19] TAKAI, I., et al. LED and CMOS image sensor based optical wireless communication system for automotive applications. IEEE Photonics Journal, 2013, vol. 5, no. 5, p. 6801418-6801418. DOI: 10.1109/JPHOT.2013.2277881

[20] NAGURA, T., et al. Tracking an LED array transmitter for visible light communications in the driving situation. In Proceedings of the 7 th International Symposium on Wireless Communication Systems (ISWCS). 2010, p. 765-769. DOI: 10.1109/ISWCS.2010.5624361

[21] NAGURA, T., et al. Improved decoding methods of visible light communication system for ITS using LED array and high-speed camera. In Proceedings of the 71st IEEE Vehicular Technology Conference (VTC 2010-Spring). 2010, p. 1-5. DOI: 10.1109/VETECS.2010.5493958

[22] KINOSHITA, M., et al. Motion modeling of mobile transmitter for image sensor based I2V-VLC, V2I-VLC, and V2V-VLC. In Proceedings of the IEEE Globecom Workshops (GC Wkshps). 2014, p. 450-455. DOI: 10.1109/GLOCOMW.2014.7063473

[23] KOMINE, T., NAKAGAWA, M. Fundamental analysis for visiblelight communication system using LED lights. IEEE transactions on Consumer Electronics, 2004, vol. 50, no. 1, p. 100-107. DOI: 10.1109/TCE.2004.1277847

[24] CAHYADI, W. A., et al. Efficient road surface detection using visible light communication. In Proceedings of the Seventh International Conference on Ubiquitous and Future Networks. 2015, p. 61-63. DOI: 10.1109/ICUFN.2015.7182498

[25] LUO, P., et al. Performance analysis of a car-to-car visible light communication system. Applied Optics, 2015, vol. 54, no. 7, p. 1696-1706. DOI: 10.1364/AO.54.001696

[26] ROBERTS, R. D. Undersampled frequency shift ON-OFF keying (UFSOOK) for camera communications (CamCom). In Proceedings of the 22nd Wireless and Optical Communication Conference (WOCC). 2013, p. 645-648. DOI: 10.1109/WOCC.2013.6676454 
[27] LUO, P., et al. Undersampled phase shift ON-OFF keying for camera communication. In Proceedings of the Sixth International Conference on Wireless Communications and Signal Processing (WCSP). 2014, p. 1-6. DOI: 10.1109/WCSP.2014.6992043

[28] UYSAL, M., et al. Optical Wireless Communications: An Emerging Technology. Springer, 2016. ISBN: 9783319302010

[29] WILKINS, A., VEITCH, J., LEHMAN, B. LED lighting flicker and potential health concerns: IEEE standard PAR1789 update. In Proceedings of the IEEE Energy Conversion Congress and Exposition (ECCE). 2010, p. 171-178. DOI: 10.1109/ECCE.2010.5618050

[30] WU, L. C., TSAI, H. M. Modeling vehicle-to-vehicle visible light communication link duration with empirical data. In Proceedings of the IEEE Globecom Workshops (GC Wkshps). 2013, p. 1103-1109. DOI: 10.1109/GLOCOMW.2013.6825140

[31] PROAKIS, J. G. Digital Communications. 4th ed. McGraw-Hill, 2000. ISBN: 978-0072321111

\section{About the Authors ...}

Stanislav VÍTEK graduated at the Czech Technical University in Prague in 2002 and received Ph.D. degree from the Czech Technical University in Prague in 2008. Recently he is an assistant professor with Dept. of Radioelectronics at the Faculty of Electrical Engineering at the Czech Technical University in Prague. Member of numerous international teams focused to networks of robotic telescopes. His main research interests are signal and image processing, embedded systems, machine learning and database systems.

Jiří LIBICH received his M.Sc. from the Czech Technical University in Prague in 2009 and Ph.D. degree in Radioelectronics in 2014. Since then, he has worked as research assistant in the CTU in areas of free space optics and visible light communications.

Pengfei LUO received his Ph.D. degree from Beijing University of Posts and Telecommunications (BUPT), China in 2013. He was a research fellow of Department of Physics and Electrical Engineering, Northumbria University, UK (12/2013-10/2014), and a project assistant in BUPT (11/2014-03/2016). Currently, he is a research engineer at Research Department of HiSilicon, Huawei Technologies Co., Ltd,. His research interests include optical camera communication, indoor positioning technology.

Stanislav ZVÁNOVEC a full professor and deputy-head of the Department of Electromagnetic Field, Faculty of Electrical Engineering, CTU. He leads Free-space and fiber optics team at CTU, was vice-chair of WG1 of EU COST project IC1101 OPTICWISE, holder of several projects. Published more than 170 papers, several books and book chapters, he is editor of book Visible Light Communications: Theory and Applications. His main research interests are wireless optical communications, visible light communications and optical fiber technologies.

Zabih GHASSEMLOOY A Research Fellow, City University, U.K (87). Sheffield Hallam University, UK (882004), University of Northumbria Newcastle, UK (2004), Chinese Academy of Science President's International Fellowship (2016). Published 695 papers (259 journals and 6 books). Research interests optical wireless communications, free space optics, and visible light communications. $\mathrm{He}$ is the fellow of the IET, a senior member of IEEE and a member of OSA.

Navid Bani HASSAN received the B.Sc. and the M.Sc. degree in electrical engineering from the Ferdowsi University of Mashhad in 2012 and Tarbiat Modares University, Tehran, in 2015, respectively. He is currently pursuing the Ph.D. degree at Northumbria University at Newcastle, Newcastle upon Tyne, UK. His research interests include car-to-car optical communications, free space optics, underwater optical communications, and digital signal processing. 